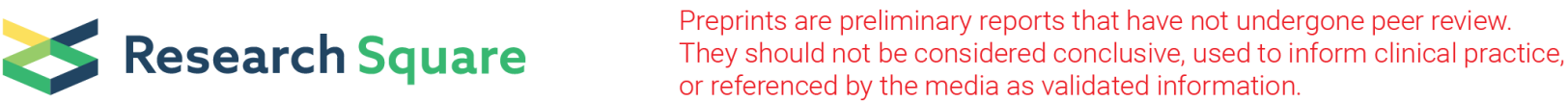

\section{MALAT-1/p53/miR-155/miR-146a ceRNA circuit tuned by Methoxylated Quercitin Glycoside Alters Immunogenic and Oncogenic Profiles of Breast cancer}

\section{Mustafa Abdel-Latif}

German University in Cairo

Ahmed Riad

German University in Cairo

Raghda Ashraf Soliman

German University in Cairo

Aisha Elkhouly

German University in Cairo

Heba Nafae

German University in Cairo

Mohamed Zakaria Gad

German University in Cairo

Amira Abdel Motaal

Cairo University Faculty of Pharmacy

Rana Ahmed Youness ( $\nabla$ rana.youness21@gmail.com )

University of Hertfordshire School of Life and Medical Sciences https://orcid.org/0000-0001-62687942

\section{Research Article}

Keywords: Cleome droserifolia, Breast cancer, TP53, miR-155, MALAT-1, Natural Killer cells, Tumor microenvironment

Posted Date: November 2nd, 2021

DOl: https://doi.org/10.21203/rs.3.rs-986101/v1

License: @ (i) This work is licensed under a Creative Commons Attribution 4.0 International License. Read Full License 
Version of Record: A version of this preprint was published at Molecular and Cellular Biochemistry on February 7th, 2022. See the published version at https://doi.org/10.1007/s11010-022-04378-4. 


\section{Abstract}

Triple Negative Breast Cancer (TNBC) is one of the most aggressive and hot BC subtypes. Our research group has recently shed the light onto the utility of natural compounds as effective immunotherapeutic agents. The aim of this study is to investigate the role of a methoxylated quercitin glycoside (MQG) isolated from Cleome droserifolia in harnessing TNBC progression and tuning the tumor microenvironment and natural killer cells cytotoxicity. Results showed that MQG showed highest potency in repressing cellular proliferation, colony forming ability, migration and invasion capacities. Mechanistically, MQG was found to modulate a circuit of competing endogenous RNAs where it was found to reduce the oncogenic MALAT-1 IncRNA and induced TP53 and its downstream miRNAs; miR-155 and miR-146a. In turn, this lead to alteration in several downstream signaling pathways such as nitric oxide machinery, natural killer cells through inducing the expression of its activating ligands such as MICA/B, ULBP2, CD155 and ICAM-1 and trimming of the immune suppressive cytokines such as TNF-a and IL-10. In conclusion, this study shows that MQG act as a compelling anti-cancer agent repressing TNBC hallmarks, activating immune cell recognition and alleviating the immune suppressive tumor microenvironment experienced by TNBC patients.

\section{Introduction}

By the end of 2020, the number of women diagnosed with breast cancer (BC) reached 7.8 million worldwide, ranking it as the most prevalent malignancy among females [1]. One of the most influential factors in terms of $\mathrm{BC}$ survival rates is the molecular subtyping of tumor. Hormone receptor positive $\left(\mathrm{HR}^{+}\right)$ $\mathrm{BC}$ correlates to the most forgiving prognoses followed by the human epidermal growth factor receptor 2 positive (HER2 ${ }^{+}$) BC subtype [2]. Bleaker still is the onset of triple negative breast cancer (TNBC) which is characterized by the lack of hormone receptors as well as HER2, which are powerful therapeutic targets within the context of the former two BC subtypes [3-5]. It is this challenge that has prompted immunotherapy as the method of choice when dealing with TNBC, whereby a variety of immune checkpoint blockers are in late stages of clinical trials [6]. These agents do, however, harbor with them a number of serious and potentially fatal side effects [7-9]. In light of this, our research group has previously demonstrated that natural compounds could be utilized in an immunotherapeutic capacity, possibly providing a means to avoid the side effects of synthetic chemotherapeutic agents [10-12]. Quercetin-3- meth $\otimes y$ - 3 - $O$ - (4'-acetylrhamnoside)-7-0-a-rhamnoside (a methoxylated quercetin glycoside (MQG) has been previously isolated by our group from the Egyptian medicinal shrub Cleome droserifolia $[13,14]$. MQG showed highly inducing effects on the tumor suppressor triad TP53, miR-15a, and miR-16 expression levels with potent antitumor activity in liver cancer cell lines [14]. However, its impact on TNBC progression has never been investigated

One of the most well-defined aspects of cancer is the myriad of epigenetic events associated with it [15]. A recent layer of complexity has been added to the epigenetic circuit tuning $B C$ progression which is the association of long non-coding RNA (IncRNA) in the regulation of fundamental BC hallmarks and downstream targets [16-18]. MALAT-1 is an oncogenic IncRNA that has been validated to modulate BC 
progression. However, its role on immunological profile has rarely been investigated. The dysfunction of the TP53 gene in particular brings about the dysregulation of a series of modulatory microRNAs (miRNAs) which in turn shepherd the mutant cell towards an immortal malignant phenotype [19-21]. Two of the most particularly influential players in this scenario are miR-155-5p and miR-146a-5p. MiR155-5p and miR-146a-5p are notorious for their roles as immunostimulant, or pro-inflammatory miRNAs [22-24] and hence are relevant in eliciting a proper immune surveillance response against the malignant transformation process. Within the tumor immune microenvironment (TIME), miR-155-5p and miR-146a$5 p$ was found to alter cytotoxic $T$ cells activity. However, their role in altering the recognition of BC cells towards the innate arm of the immune system and its impact on the cytokine storm at the TIME is yet to be investigated. Therefore, the aim of this experiment is to unravel the impact of MQG on the oncological or the immunological profiles of $\mathrm{BC}$ cells and to further investigate the underlying molecular mechanism underneath such alterations

\section{Materials And Methods}

\section{Cell culture and treatment}

Different cancer cell lines such as TNBC cell lines, MDA-MB-231, $\mathrm{HR}^{+} \mathrm{BC}$ cell lines, MCF-7 and HCC cell lines Hep-G2 and Huh7 cells were obtained from ATCC and Vacsera, Egypt. Adherent cells were cultured in DMEM (Lonza, Switzerland) media as previously described [14, 25, 26]. The respective compounds (13) were isolated from C.droserifolia as previously described our group [14] and shown in Figure 1. Stock solutions were prepared as $250 \mu \mathrm{M}$ stock solutions in $0.1 \%$ DMSO in culture media. Effective concentrations ranging from $1 \mu \mathrm{M}$ to $200 \mu \mathrm{M}$, depending on the experimental setup, were prepared and used to treat different cancer cells seeded in 96 -well or 24-well plates. Cells were treated for $24-96 \mathrm{~h}$ in normal growth conditions $\left(37^{\circ} \mathrm{C}\right.$ in $5 \% \mathrm{CO}_{2}$ atmosphere). 5 -Fluorouracil was used as a positive control in this study. In all experiments, cells used as a control were labeled as vehicle control and were exposed to $0.1 \%$ DMSO in culture media as previously described [10, 27-29].

\section{Cellular viability and proliferation experiments}

3-(4, 5-dimethylthiazol-2-yl)-2,5-diphenyltetrazolium bromide (MTT) reagent was used in the cellular viability experiments. BC cells $(10,000)$ were seeded in $200 \mu \mathrm{L}$ full media in a 96-well plate. Forty-eight $\mathrm{h}$ post-transfection, media was replaced by $20 \mu \mathrm{L}$ working solution. After $6 \mathrm{~h}$, the absorbance of the formed purple formazan crystals, solubilized in $200 \mu \mathrm{L}$ lysis buffer, was measured [14, 25, 26, 30]. For the cellular proliferation experiments, bromo-deoxyuridine (BrdU) incorporation assay was used. $\mathrm{BC}$ cells were seeded into black 96 -well plates at a cell density of $5 \times 10^{4}$ cells/well. According to the Cell Proliferation ELISA kit protocol (Roche Applied Science, Penzberg, Germany), BC cells were incubated with BrdU for $4 \mathrm{~h}$, then fixed for 30 min using Fix-Denate and finally incubated with Anti-BrdU POD for $90 \min [14,25,26,30]$. All experiments were performed in triplicates and repeated three times or more.

\section{Cellular migration and invasion}


BC migration capacity was assessed using the wound-healing/scratch assay. Treated cells were left to grow to a confluency of $90-95 \%$. Post-treatment, 3 scratches were performed in each well using a $10-\mu \mathrm{L}$ pipette tip. BC cells were washed using PBS and replenished with new low-serum media (1\% FBS). After $24 \mathrm{~h}$, the surface areas of the scratches were measured and wound closure was quantified with Zen2012 software $[14,25,26,30]$. While for the invasion experiments, the modified boyden chamber assay (BD Bioscience, Bedford, USA) was performed. In 24-well plates, BC cells were treated with MQG and then $6 \times 10^{4}$ cells were re-suspended in $200 \mu \mathrm{L}$ low-serum media ( $\left.1 \% \mathrm{FBS}\right)$ and were seeded in the upper well. Yet, the lower well contained high-serum media (20\% FBS). Cells were washed from the upper surface using a cotton swab $8 \mathrm{~h}$ after seeding. Then the invaded cells were fixed and stained using $1 \%$ crystal violet (Sigma Chemical Co., California, USA) and counted under an inverted light microscope. All experiments were performed in triplicate and repeated three times or more [14, 25].

\section{Colony-forming assay}

For the colony forming experiment, treated cells were harvested and seeded post treatment in 6-well plate at 800 cells/well. BC cells were incubated in full DMEM under normal conditions $\left(37^{\circ} \mathrm{C}\right.$ and $\left.5 \% \mathrm{CO}_{2}\right)$ for 23 weeks. Colonies were fixed using $6 \%$ glutaraldehyde, stained by $0.05 \%$ crystal violet and then counted $[14,25]$.

\section{Cell cycle analysis}

Expression vectors containing response elements for vital cell cycle proteins such as TP53 (pp53-TA-Luc), c-Myc (pMyc-TA-Luc), RB (pRB-TA-Luc), E2F (pE2F-TA-Luc; Clontech, France) were used. Similarly, BC cells were treated with pLuc vector containing an unspecific binding site (Clontech, France). BC cells were seeded and transfected with the respective vectors using Superfect Transfection Reagent (Qiagen, Germany) according to the manufacturer's protocol. After $24 \mathrm{~h}$ post-transfection of the plasmid DNA, cells were treated with MQG. After $72 \mathrm{~h}, \mathrm{BC}$ cells were then lysed and luciferase expression/luminescence measurement was quantified using Steady-GLO Luciferase Kit (Promega, Germany) according to the manufacturer's instructions. Luminescence was plotted as \% luciferase activity relative to cells transfected with the vector alone. Unspecific luminescence detected by the reagents and the empty pLuc vector (baseline luminescence) was subtracted from all values before plotting as previously illustrated [4, 25].

\section{Total RNA and miRNAs extraction}

Total RNA and miRNAs were isolated using Biozol RNA extraction kit. Extracted RNA was then quantified spectrophotometrically. RNA integrity was examined by 18 s rRNA bands detection on $1 \%$ agarose gel electrophoresis. RNA samples with 260/280 optical density $>2$ were excluded $[14,25,26,30]$.

\section{Quantitative real-time PCR analysis}

Reverse transcription and relative expression quantification of MALAT-1, NOS2, NOS3, MICA, MICB, ULBP2, CD155, ICAM-1, TNF-a, IL-10 and $\beta$-actin mRNAs were performed. High-Capacity cDNA Reverse Transcription Kit ( $\mathrm{BBI}$, California, USA) was used according to the manufacturer's instruction for the 
reverse transcription process. While for the extracted miRNAs TaqMan MicroRNA Reverse Transcription Kit ( $\mathrm{ABI}$, California, USA) was used using specific primers for hsa-miR-155, has-miR-146a and RNU6B. Concerning the relative expression analysis of all the targets and housekeeping genes used in this study TaqMan Real-Time q-PCR on StepOne ${ }^{\mathrm{TM}}$ Systems (ABI, California, USA) was used. Relative expression calculations were performed using the $2^{-\Delta \Delta C t}$ method. All PCR reactions were done in triplicates and repeated 3 times or more $[14,25,26,30-32]$.

\section{Quantification of NO Production}

NO production was measured using Griess reagent assay (Promega, USA) according to the manufacturer protocol $[4,25]$. Briefly, $50 \mu \mathrm{L}$ of cells' supernatant were mixed with $50 \mu \mathrm{L}$ sulfanilamide solution and incubated for $10 \mathrm{~min}$. Then, another $50 \mu \mathrm{L}$ of N-1-napthylethylenediamine dihydrochloride (NED) solution is added and absorbance was measured at $540 \mathrm{~nm}$ using Wallac 1420 Victor 2 Multilabel Counter (Perkin Elmer, USA). Experiments were performed in triplicates and repeated 3 times or more $[16,25]$.

\section{NK cell isolation}

Peripheral blood mononuclear cells (PBMCs) were separated from peripheral blood of healthy donors upon their written informed consent using Ficoll-Hypaque centrifugation (Axis-Shield PoC AS, Norway). NK cells were then enriched by negative selection using a MACS NK cell isolation kit (Miltenyi Biotec, Cologne, Germany). Enriched NK cell populations were 96.9\% CD56/CD3 and 0.8\% CD3 positive, assessed by flow cytometry $[26,30,33,34]$.

\section{Lactate dehydrogenase (LDH) assay}

Treated BC cells were seeded in a 96-well plate at a cell density of 15,000 cells/well. After $2 \mathrm{~h}$, primary NK cells were added to the target $\mathrm{BC}$ cells at a 5:1 effector to target ratio (E:T) and incubated for $8 \mathrm{~h}$. Later, the lactate dehydrogenase (LDH) activity assay kit (MAK066-1K1-Sigma-Aldrich, St. Louis, MO, USA) was used to measure the in-vitro NK cells cytotoxic potential following the manufacturer's instructions. The lysis \% was calculated according to the following equation: \% cytotoxicity = (target maximum release experimental release)/(target maximum release) $\times 100$. The experiment was done in triplicate and repeated more than 3 times $[25,26,30,33,35]$

\section{Statistical analysis}

Data is presented as mean \pm standard error of the mean (SEM) for 3 different experiments. Nonparametric unpaired student-t-test was executed to compare between every two independent groups. Oneway analysis of variance with post hoc analysis was adopted for multiple comparisons. P-value of $<0.05$ was considered statistically significant, and the threshold of significance is denoted by $*=p<0.05$, $\star \star p<0.01, * \star \star=p<0.001, * \star \star \star *=p<0.0001$. Data was analyzed using GraphPad Prism 8.2.1 software as previously described [10].

\section{Results}




\section{Preferential selectivity of flavonol glycosides towards MDA- MB-231 TNBC cell lines}

The cytotoxic profiles of flavonol glycosides isolated from $C$. droserifolia (Compounds 1-3) were screened against different cancer cell lines. Flavonol glycosides showed a preferential selectivity towards the TNBC cell lines, MDA-MB-231 when compared to $\mathrm{HR}^{+} \mathrm{BC}$ cells and $\mathrm{HCC}$ cell lines as shown in Table 1 and Figure 2. Therefore, the other functional analysis experiments were performed on MDA-MB-231 TNBC cells. The $\mathrm{IC}_{50}$ values of the respective compounds were calculated using the corresponding doseresponse curves of each compound in each cell line after 3 days of treatment.

Table 1

$\mathrm{IC}_{50}$ values of falvonol glycosides (Compounds 1-3) isolated from C.droserifloia aganist different cancer cell lines

\begin{tabular}{|lllll|}
\hline $\begin{array}{l}\text { Flavonol } \\
\text { glycoside }\end{array}$ & $\begin{array}{l}\mathrm{IC}_{50} \text { in Hep-G2 } \\
\text { cells }\end{array}$ & $\begin{array}{l}\mathrm{IC}_{50} \text { in Huh-7 } \\
\text { cells }\end{array}$ & $\begin{array}{l}\mathrm{IC}_{50} \text { in MCF-7 } \\
\text { cells }\end{array}$ & $\begin{array}{l}\mathrm{IC}_{50} \text { in MDA-MB-231 } \\
\text { cells }\end{array}$ \\
\hline Compound 1 & $28 \pm 1.63 \mu \mathrm{M}$ & $50 \pm 2.25 \mu \mathrm{M}$ & $46.5 \pm 1.05 \mu \mathrm{M}$ & $21.7 \pm 1.75 \mu \mathrm{M}$ \\
\hline Compound 2 & $18 \pm 1.70 \mu \mathrm{M}$ & $41 \pm 3.68 \mu \mathrm{M}$ & $38 \pm 3.12 \mu \mathrm{M}$ & $12 \pm 2.41 \mu \mathrm{M}$ \\
\hline Compound 3 & $35 \pm 3.27 \mu \mathrm{M}$ & $60 \pm 2.94 \mu \mathrm{M}$ & $51.5 \pm 1.85 \mu \mathrm{M}$ & $30.3 \pm 1.61 \mu \mathrm{M}$ \\
\hline
\end{tabular}

Impact of serial dilutions of flavonol glycosides isolated fromC. droserifoliaon MDA-MB-231 cellular viability and proliferation rates

Serial dilutions of flavonol glycosides $(1-10 \mu \mathrm{M})$ were prepared and applied to MDA-MB-231 cells for $72 \mathrm{~h}$. Compounds (1-3) showed a concentration dependent reduction in the cellular viability (Figure 3a-c) and cellular proliferation rate (Figure 3d-f). Compound 2 was the most potent compound (lowest $\mathrm{IC}_{50}$ as indicated in Table 1) and showed the most repressing effects on MDA-MB-231 cellular proliferation when compared to compounds 1 and 3 .

Impact of flavonol glycosides isolated from C. droserifolia on MDA-MB-231 colony forming ability, migration and invasion capacities

To investigate the long-term effects of serial dilutions of $C$. droserifolia flavonol glycosides (1-3) on MDAMB-231 cell lines, anchorage-independent growth assay was performed. In a similar pattern to cellular viability and proliferation assays, compounds (1-3) showed a concentration dependent reduction in the clonogenic properties also with compound 2 (MQG) showing the most potent inhibitory impact as shown in (Figure 4a-c). It was tempting to further investigate the impact of $C$. droserifolia flavonol glycosides, Compounds (1-3), on the migration and invasion capacities of MDA-MB-231 cells. Compounds (1-3) (IC $\mathrm{I}_{50}$ values) showed a significant attenuation of wound closure when compared to vehicle control cells. In a similar pattern to cellular viability and anchorage-independent growth assays, Compound 2 (MQG) 
showed the lowest \% closure of the wound $(26.36 \pm 1.268)$ if compared to Compound $1(48.49 \pm 4.187)$ and Compound 3 (48.24 \pm 3.645$)$ (Figure $4 d$ ).

\section{Impact of Methoxylated Quercetin Glycoside on cell cycle proteins}

After validating the potential selective anticancer activity of MQG in halting the oncogenic profile of human BC cells effectively and being non-toxic to human normal cells (Supplementary Figure 1), our aim was extrapolated to unravel the mechanism by which MQG could harness BC progression. For that reason, vital cell cycle proteins were screened such as the tumor suppressor TP53, the oncogenic protein cMyc and the RB/E2F complex proteins. The results showed that MQG led to a significant elevation in TP53 protein levels $(P<0.0001)$. However, it had no effect on c-Myc and RB/E2F complex activity (Figure $5 a-d)$.

\section{Impact of Methoxylated Quercetin Glycoside on ncRNAs circuit around TP53}

Then, it was interesting to further unravel the machinery downstream/upstream the TP53. TP53 is a wellknown upstream regulator for an array of miRNAs previously validated by our group [4]. Nonetheless, it was recently reported that MALAT-1 IncRNA acts as a direct upstream regulator for TP53 [36]. On the other hand, miR-155 and miR-146a are reported to act as downstream miRNAs to TP53 and have dual role in tuning the oncological and immunological profiles. For that reason, screening of MALAT-1, miR155 and miR-146a was performed in treated MDA-MB-231 cells by $\sim I_{50}$ value $(10 \mu \mathrm{M})$ of MQG. The results showed that the oncogenic MALAT-1 IncRNA has been significantly reduced $(P<0.001)$ while a marked indication of miR-155 $=(P=0.0002)$ and miR-146a $(P=0.0026)$ levels were observed (Figure 6); building up a novel axis MALAT-1/TP53/miR-155/miR-146a drawn downstream MQG in TNBC cells.

\section{Impact of Methoxylated Quercetin Glycoside on NO machinery system}

Furthermore, nitric oxide (NO) has been validated as an important cytokine at the TIME and at the same time has an indisputable role in altering the oncological profile of BC cells. Interestingly, the NO synthesizing enzymes (NOS2 and NOS3) are validated targets for miR-155 and miR-146a. Therefore, in an attempt to draw the full axis downstream MQG in TNBC cells, the impact of MQG on NO machinery system was probed. Interestingly, MQG resulted in a marked repression of NOS2 ( $P=0.0030)$, NOS3 $(P=0.0005)$ mRNA levels and consequently a marked reduction in the NO produced from MDA-MB-231 $(P=0.0020)$ (Figure 7).

\section{Impact of Methoxylated Quercetin Glycoside on NK cells cytotoxicity and tumor microenvironment}


After validating the alteration of MALAT-1/TP53/miR-155/miR-146a machinery upon MQG treatment for MDA-MB-231 cell, we turned our attentions towards miR-155 and miR-146a's immunostimulant roles in the context of BC. It was essential to investigate the impact of MQG on miR-155 and miR-146a immunerelated validated targets such as NK cells activating ligands (MICA/B, ULBP2, CD155, ICAM-1), known to be markedly down-regulated in MDA-MB-231 cells, and vital immune-inhibitory cytokines (TNF- $a$, IL-10) known to have a dominant role in potentiating the immune-suppressive microenvironment in $\mathrm{BC}$ patients. MQG resulted in a marked induction of MICA ( 8 folds. $P=0.0158$ ), MICB (10 folds, $P<0.0001)$, ULBP2 (3 folds, $P<0.0001$ ), $C D 155$ (2 folds, $P=0.0038$ ), ICAM-1 ( 3 folds, $P=0.0188$ ) and significant reduction of immune suppressive cytokines TNF- $\alpha(P=0.0228)$ and IL-10 $(P=0.0006)$. Collectively, this resulted in an induction in primary NK cells cytotoxicity $(P=0.0001)$ (Figure 8a-h).

\section{Discussion}

The current study sheds the light onto a novel crosstalk between ncRNAs building up a novel ceRNAs circuit and their respective preys (targets). MQG was found to have the lowest $\mathrm{IC}_{50}$ in repressing the proliferation and viability of MDA-MB-231 TNBC cell lines. TNBC patients are the least fortunate if compared to other $\mathrm{BC}$ subtypes. The lack of therapeutic targets renders its patients especially needful of additional treatment options. Hence; TNBC patients comprised the chief scope of our research. Mechanistically, it was important to unravel the mechanism by which MQG acts as a potent selective anti-cancer agent against MDA-MB-231 cells. TP53 is the chief tumor suppressor protein and the most fundamental orchestrator of apoptosis and cell cycle arrest [37]. TP53 is markedly down regulated in TNBC patients and cell lines $[34,38,39]$. For that reason, TP53 was our primary target to unravel MQG molecular mechanism in TNCB cell lines. Consistent with our previous study [14], the results showed that TP53 transcript and protein levels are induced by MQG in MDA-MB-231 cells. In the current study, we further assayed the protein levels of the alternative apoptotic genes E2F, RB, and C-MYC, all of which showed no increase upon treatment with MQG. Taken together, these results suggest that the significant cytotoxicity of MQG is partially mediated by $\mathrm{p} 53$. Then, it was intriguing to unravel the ncRNAs circuit revolving around the TP53. MALAT-1 is an oncogenic IncRNA that was recently reported to modulate TP53 in mice but it never been investigated in BC. Nonetheless, miR-155 and miR-146a were recently reported to act as ceRNA molecules with MALAT-1 and at the same time they are drawn downstream TP53 in several cellular context $[22,40]$. Yet, such novel axis has never been investigated in TNBC cells. For this reason, we extended our view towards the variety of implications p53 induction would entail. The results showed a repression of MALAT-1 concomitant with a marked repression with miR-155 and miR146a in MQG treated TNBC cells; Thus building up a novel ceRNA axis orchestrated by TP53 drawn downstream MQG in MDA-MB-231 cells. The clear increase in the expression levels of these two miRNAs witnessed in our study likely supports the hypothesis of an induction mechanism facilitated by the initial modulation of MALAT-1/p53 axis. This goes in line with previous studies highlighting the potent effect of quercetin derivatives on modulating the expression of ncRNAs such as H19 IncRNA, miR-486-5p, miR548a, miR-20a [27-29, 35] 
Further downstream still is the observation of NOS2 and NOS3 down-regulation- 2 validated target genes of miRNA-155 and miRNA-146a simultaneously [41, 42]. Oft dysregulated in cancer, the NO machinery (i.e. NOS2/NOS3) is highly implicated in driving tumor migration, angiogenesis, and mutation through radical-based DNA damage [43]. Indeed, high NOS activity is a significant predictor for poor prognosis among TNBC patients $[44,45]$. Our results pertaining to the general mitigation of NO machinery upon MQG treatment thus demonstrate an additional facet of its antitumor activity.

The emergence of immunotherapy during the past decade brought about an entirely new line of thinking in the landscape of cancer therapy-prompting a widespread consensus of the immune system as the future of the oncological field. With this in mind, we chose to furthermore investigate MALAT- $1 / \mathrm{p} 53 / \mathrm{miR}$ $155 /$ miR-146a axis' significant role in modulating the innate immune recognition. Accordingly, our study showed significant increases in expression of the NK cell ligands MICA/B, ULBP2, CD155, and ICAM-1- as downstream targets for the ceRNA molecules MALAT-1/miR-155/miR-146a [46-48]. These ligands, in a normal context, act as signals for NK cell cytotoxicity during cellular stress. As a result, one mechanism of carcinogenic evolution is the shedding of these ligands, which are found to be especially downregulated in aggressive BC [49] such as TNBC. Their induction in MDA-MB-231 upon treatment with MQG and subsequent increase in NK-mediated cytotoxicity strongly alludes to a counteraction of this immune evasion mechanism. MQG administration also exhibited a significant down-regulation of IL-10, an inhibitory cytokine to $\mathrm{NK}, \mathrm{CD}^{+}$, and dendritic cells among others and a reportedly negative prognostic factor in $B C[50,51]$. In doing so, a tentative but promising window is opened towards an entirely new addition to the immunotherapeutic arsenal- shifting the focus from the currently dominant adaptive immunity-based agents for which resistance is growing and intense side effects are reported [8, 52-54]. MQG's derivation from natural sources moreover represents a toxicologically safe and potentially much cheaper alternative to synthetic immunotherapeutic agents.

Curiously, we observed a concomitant downregulation of TNF-a upon MQG administration, despite reports of a positive correlation between miR-155 and the cytokine $[55,56]$. High TNF-a production contributes to an inflamed, immunosuppressive TIME and has in fact been reported to mitigate TNBC progression in vitro when knocked out [57]. Although somewhat counterintuitive, the downregulation of TNF-a witnessed in our study hence signifies another favorable modulation to the TIME imposed by MQG. It must be taken into account that, in inducing p53, the expression of several miRNAs apart from the ones we have tested is overwhelmingly likely to have been affected. The suppression of TNF- $a$ therefore could be the result of a differential miR pathway such as miR-140-5p/miR-181a-5p $[58,59]$

In conclusion, the current study probe a detailed molecular mechanism detailing the impact of MQG on the oncological and the immunological profiles of TNBC cells. Furthermore, this study sheds the light onto a novel selective multifaceted anticancer immunotherapeutic nutraceutical that holds a great potential for TNBC patients. This study also demonstrates a novel ceRNA circuit MALAT-1/miR-155/miR$146 a$ orchestrated by p53 in TNBC cells and thus possesses potent effectiveness against TNBC progression and aggressiveness and immune suppressive nature as summarized in Figure 9. 


\section{Abbreviations}

Breast Cancer $\quad B C$

Cluster of differentiation 155

CD155

Interleukin-10

IL-10

Intracellular adhesion molecule-1

ICAM-1

MHC class I-related chain A

MICA

MHC class I-related chain B

MICB

microRNA

miRNA/miR

Natural Killer cells

NK cells

Triple negative breast cancer

TNBC

Tumor necrosis factor-alpha

TNF-a

UL-16 binding protein

ULBP2

\section{Declarations}

\section{Acknowledgment}

Not available

\section{Conflict of Interest}

All authors declare no conflict of interest

\section{Funding Agency}

This study was not supported by any funding agency

\section{Ethical Statement}

This study compiles with all Ethical Standards. The current study does not include any human particpants or animals so informed consents are not applicable

\section{Data Availability}

Data is available from the corresponding author based on a reasonable request 


\section{Author's Contribution}

Abdel-Latif M. and Youness R.A. contributed in most of the practical work and wrote the mansucript. Riad A., Soliman, R., El-khouly A., Nafae H. contributed in the practical work. Gad M.Z., Motaal A. co-supervised the work. Youness R.A. conceived the original idea, designed the experimental setup, supervised the work All authors discussed the results and contributed to the final manuscript.

\section{References}

1. Siegel, R.L., K.D. Miller, and A. Jemal, Cancer statistics, 2020. CA Cancer J Clin, 2020. 70(1): p. 730.

2. O'Brien, K.M., et al., Intrinsic Breast Tumor Subtypes, Race, and Long-Term Survival in the Carolina Breast Cancer Study. Clinical Cancer Research, 2010. 16(24): p. 6100-6110.

3. Dent, R., et al., Triple-negative breast cancer: clinical features and patterns of recurrence. Clin Cancer Res, 2007. 13(15 Pt 1): p. 4429-34.

4. Youness, R.A., et al., The long noncoding RNA sONE represses triple-negative breast cancer aggressiveness through inducing the expression of miR-34a, miR-15a, miR-16, and let-7a. J Cell Physiol, 2019. 234(11): p. 20286-20297.

5. El-Layeh, R.A., et al., 36PStructural diversity of the cardenolide calotropin renders it as a targeted therapy for harnessing TNBC progression through tuning nitric oxide (NO) levels. Ann Oncol, 2019. 30(Supplement_1).

6. Katz, H. and M. Alsharedi, Immunotherapy in triple-negative breast cancer. Med Oncol, 2017. 35(1): p. 13.

7. Beck, K., et al., Enterocolitis in Patients With Cancer After Antibody Blockade of Cytotoxic TLymphocyte-Associated Antigen 4. Journal of Clinical Oncology, 2006. 24(15): p. 1-16.

8. van der Vlist, M., et al., Immune checkpoints and rheumatic diseases: what can cancer immunotherapy teach us? Nat Rev Rheumatol, 2016. 12(10): p. 593-604.

9. Abdel-Latif, M. and R.A. Youness, Why natural killer cells in triple negative breast cancer? World J Clin Oncol, 2020. 11(7): p. 464-476.

10. Awad, A.R., et al., An acetylated derivative of vitexin halts MDA-MB-231 cellular progression and improves its immunogenic profile through tuning miR-20a-MICA/B axis. Natural product research, 2019: p. 1-5.

11. El-Layeh, R.A., et al., Structural diversity of the cardenolide calotropin renders it as a targeted therapy for harnessing TNBC progression through tuning nitric oxide (NO) levels. Annals of oncology : 
official journal of the European Society for Medical Oncology, 2019. 30 Suppl 1: p. i14.

12. Elkhouly, A., et al., miR-486-5p and miR-17-5p: Novel Immunomodulatory Non-coding RNAs Drawn Downstream 3\&\#x2032;-O-Acetylvitexin in Triple Negative Breast Cancer. European Journal of Cancer, 2020. 138: p. S70.

13. Ezzat, S.M. and A. Abdel Motaal, Isolation of New Cytotoxic Metabolites from Cleome droserifolia

Growing in Egypt. Zeitschrift für Naturforschung, 2012. 67c: p. 266-274.

14. Youness, R.A., et al., A methoxylated quercetin glycoside harnesses HCC tumor progression in a TP53/miR-15/miR-16 dependent manner. Nat Prod Res, 2018: p. 1-6.

15. Sharma, S., T.K. Kelly, and P.A. Jones, Epigenetics in cancer. Carcinogenesis, 2010. 31(1): p. 2736.

16. Nafea, H., et al., LnCRNA HEIH/miR-939-5p interplay modulates triple-negative breast cancer progression through NOS2-induced nitric oxide production. J Cell Physiol, 2021. 236(7): p. 5362-5372.

17. Youness, R.A. and M.Z. Gad, Long non-coding RNAs: Functional regulatory players in breast cancer. Noncoding RNA Res, 2019. 4(1): p. 36-44.

18. ElKhouly, A.M., R.A. Youness, and M.Z. Gad, MicroRNA-486-5p and microRNA-486-3p: Multifaceted pleiotropic mediators in oncological and non-oncological conditions. Noncoding RNA Res, 2020. 5(1): p. 11-21.

19. Abdi, J., et al., Role of tumor suppressor p53 and micro-RNA interplay in multiple myeloma pathogenesis. Journal of Hematology \& Oncology, 2017. 10: p. 11.

20. Hattori, $\mathrm{H}$., et al., p53 shapes genome-wide and cell type-specific changes in microRNA expression during the human DNA damage response. Cell Cycle, 2014. 13(16): p. 2572-2586.

21. Sandhu, R., et al., Overexpression of miR-146a in basal-like breast cancer cells confers enhanced tumorigenic potential in association with altered p53 status. Carcinogenesis, 2014. 35(11): p. 2567-75.

22. Faraoni, I., et al., miR-155 gene: A typical multifunctional microRNA. Biochimica Et Biophysica Acta-Molecular Basis of Disease, 2009. 1792(6): p. 497-505.

23. Wang, H., et al., Regulation of Human Natural Killer Cell IFN-y Production by MicroRNA-146a via Targeting the NF-KB Signaling Pathway. Front Immunol, 2018. 9: p. 293.

24. Hargreaves, B.K.V., et al. Highly efficient serum-free manipulation of miRNA in human NK cells without loss of viability or phenotypic alterations is accomplished with Trans/T-TKO. PloS one, 2020. 15, e0231664 DOI: 10.1371/journal.pone.0231664. 
25. Youness, R.A., et al., A novel role of SONE/NOS3/NO signaling cascade in mediating hydrogen sulphide bilateral effects on triple negative breast cancer progression. Nitric Oxide, 2018. 80: p. 12-23.

26. Youness, R.A., et al., Contradicting interplay between insulin-like growth factor-1 and miR-486-5p in primary NK cells and hepatoma cell lines with a contemporary inhibitory impact on HCC tumor progression. Growth Factors, 2016. 34(3-4): p. 128-40.

27. Awad, A.R., et al., An acetylated derivative of vitexin halts MDA-MB-231 cellular progression and improves its immunogenic profile through tuning miR-20a-MICA/B axis. Nat Prod Res, 2021. 35(18): $p$. 3126-3130.

28. Mekky, R.Y., et al., Epigallocatechin gallate (EGCG) and miR-548m reduce HCV entry through repression of CD81 receptor in HCV cell models. Arch Virol, 2019. 164(6): p. 1587-1595.

29. Ahmed Youness, R., et al., A methoxylated quercetin glycoside harnesses HCC tumor progression in a TP53/miR-15/miR-16 dependent manner. Nat Prod Res, 2020. 34(10): p. 1475-1480.

30. Youness, R.A., et al., MicroRNA-486-5p enhances hepatocellular carcinoma tumor suppression through repression of IGF-1R and its downstream mTOR, STAT3 and c-Myc. Oncol Lett, 2016. 12(4): p. 2567-2573.

31. Youssef, S.S., et al., PNPLA3 and IL 28B signature for predicting susceptibility to chronic hepatitis $C$ infection and fibrosis progression. Arch Physiol Biochem, 2019: p. 1-7.

32. El Din, G.S., et al., miRNA-506-3p Directly Regulates rs10754339 (A/G) in the Immune Checkpoint Protein B7-H4 in Breast Cancer. Microrna, 2020. 9(5): p. 346-353.

33. Rahmoon, M.A., et al., MiR-615-5p depresses natural killer cells cytotoxicity through repressing IGF-1R in hepatocellular carcinoma patients. Growth Factors, 2017. 35(2-3): p. 76-87.

34. Youness, R.A., et al., Targeting hydrogen sulphide signaling in breast cancer. J Adv Res, 2021. 27: p. 177-190.

35. Abdallah, R.M., et al., Hindering The Synchronization Between Mir-486-5p And H19 Lncrna By Hesperetin Halts Breast Cancer Aggressiveness Through Tuning ICAM-1. Anticancer Agents Med Chem, 2021.

36. Zhang, T., et al., MALAT1 Activates the P53 Signaling Pathway by Regulating MDM2 to Promote Ischemic Stroke. Cell Physiol Biochem, 2018. 50(6): p. 2216-2228.

37. Petitjean, A., et al., TP53 mutations in human cancers: functional selection and impact on cancer prognosis and outcomes. Oncogene, 2007. 26(15): p. 2157-65. 
38. Horigome, E., et al., Mutant TP53 modulates metastasis of triple negative breast cancer through adenosine A2b receptor signaling. Oncotarget, 2018. 9(77): p. 34554-34566.

39. Li, J.P., et al., Association of $p 53$ expression with poor prognosis in patients with triple-negative breast invasive ductal carcinoma. Medicine (Baltimore), 2019. 98(18): p. e15449.

40. Grespi, F., et al., Differential regulated microRNA by wild type and mutant $p 53$ in induced pluripotent stem cells. Cell Death \& Disease, 2016. 7(12): p. e2567-e2567.

41. Dai, R., et al., Suppression of LPS-induced Interferon-y and nitric oxide in splenic lymphocytes by select estrogen-regulated microRNAs: a novel mechanism of immune modulation. Blood, 2008. 112(12): p. 4591-4597.

42. $\mathrm{Xu}, \mathrm{C}$., et al., miR-155 regulates immune modulatory properties of mesenchymal stem cells by targeting TAK1-binding protein 2. The Journal of biological chemistry, 2013. 288(16): p. 11074-11079.

43. Jadeski, L.C., C. Chakraborty, and P.K. Lala, Role of nitric oxide in tumour progression with special reference to a murine breast cancer model. Canadian Journal of Physiology and Pharmacology, 2002. 80(2): p. 125-135.

44. Walsh, E.M., et al., Review of Triple Negative Breast Cancer and the Impact of Inducible Nitric Oxide Synthase on Tumor Biology and Patient Outcomes. Critical reviews in oncogenesis, 2016. 21(5-6): p. 333-351.

45. Garrido, P., et al., Impact of inducible nitric oxide synthase (iNOS) expression on triple negative breast cancer outcome and activation of EGFR and ERK signaling pathways. Oncotarget, 2017. 8(46): $p$. 80568-80588.

46. Lu, C., et al., miR-221 and miR-155 regulate human dendritic cell development, apoptosis, and IL12 production through targeting of p27kip1, KPC1, and SOCS-1. Blood, 2011. 117(16): p. 4293-303.

47. Cerutti, C., et al., MicroRNA-155 contributes to shear-resistant leukocyte adhesion to human brain endothelium in vitro. Fluids Barriers CNS, 2016. 13(1): p. 8.

48. Abdel-Latif, M., et al., 23P - A new quercetin glycoside enhances TNBC immunological profile through TP53/miR-155/MICA/ULBP2. Annals of Oncology, 2019. 30: p. vii7-vii8.

49. de Kruijf, E.M., et al., NKG2D ligand tumor expression and association with clinical outcome in early breast cancer patients: an observational study. BMC Cancer, 2012. 12: p. 24.

50. Llanes-Fernández, L., et al., Relationship between IL-10 and tumor markers in breast cancer patients. Breast, 2006. 15(4): p. 482-9. 
51. Chavey, C., et al., Oestrogen receptor negative breast cancers exhibit high cytokine content. Breast Cancer Res, 2007. 9(1): p. R15.

52. Jenkins, R.W., D.A. Barbie, and K.T. Flaherty, Mechanisms of resistance to immune checkpoint inhibitors. Br J Cancer, 2018. 118(1): p. 9-16.

53. O'Donnell, J.S., et al., Resistance to PD1/PDL 1 checkpoint inhibition. Cancer Treatment Reviews, 2017. 52: p. 71-81.

54. Downey, S.G., et al., Prognostic factors related to clinical response in patients with metastatic melanoma treated by CTL-associated antigen-4 blockade. Clin Cancer Res, 2007. 13(22 Pt 1): p. 6681-8.

55. Pedersen, I.M., et al., Onco-miR-155 targets SHIP1 to promote TNFa-dependent growth of B cell lymphomas. 2009. 1(5): p. 288-295.

56. Bala, S., et al., Up-regulation of MicroRNA-155 in Macrophages Contributes to Increased Tumor Necrosis Factor alpha (TNF alpha) Production via Increased mRNA Half-life in Alcoholic Liver Disease. Journal of Biological Chemistry, 2011. 286(2): p. 1436-1444.

57. Pileczki, V., et al., TNF-a gene knockout in triple negative breast cancer cell line induces apoptosis. Int J Mol Sci, 2012. 14(1): p. 411-20.

58. Zhu, J., et al., TNF-a mRNA is negatively regulated by microRNA-181a-5p in maturation of dendritic cells induced by high mobility group box-1 protein. Scientific Reports, 2017. 7(1): p. 12239.

59. Zhu, T.T., et al., MicroRNA-140-5p targeting tumor necrosis factor-a prevents pulmonary arterial hypertension. J Cell Physiol, 2019. 234(6): p. 9535-9550.

\section{Supplementary Figure}

Supplementary Figure 1 is not available with this version

\section{Figures}




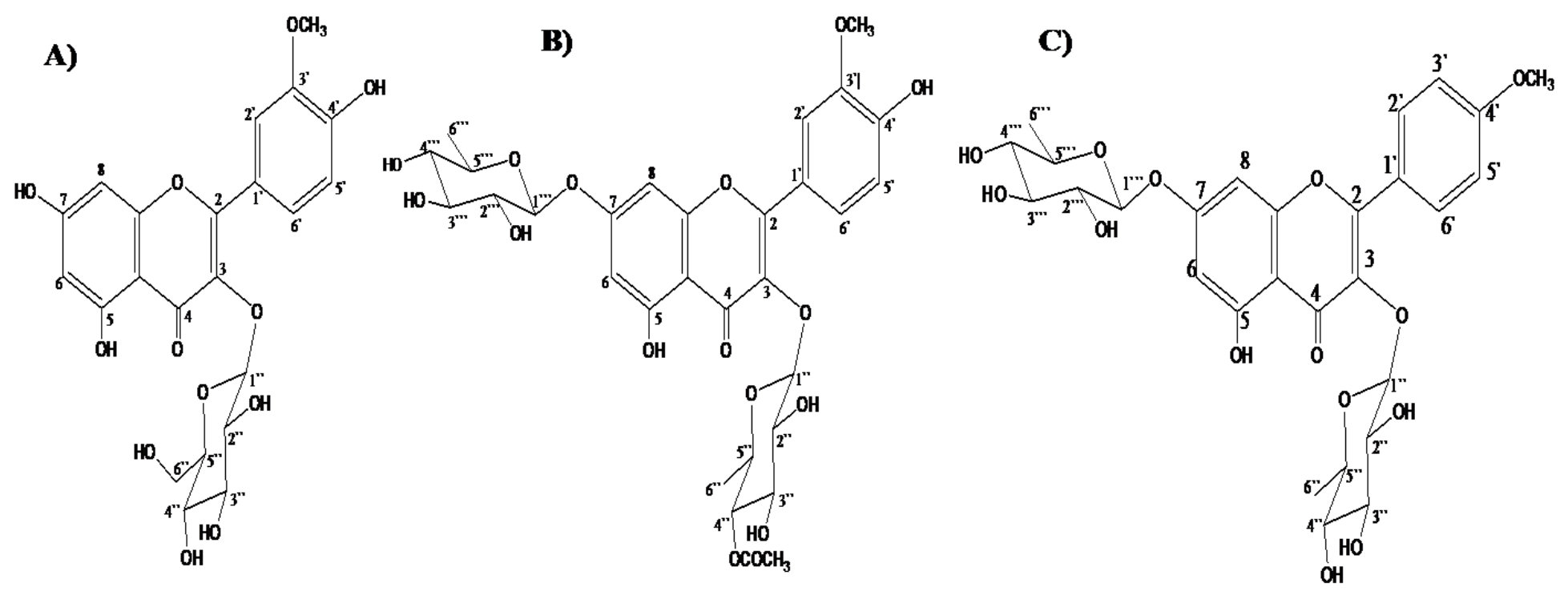

Figure 1

Flavonol glycosides isolated from C.droserifolia Chemical structure of 3 flavonol glycosides isolated from C.droserifolia (A) Compound (1), isorhamnetin-3

-meth $\otimes y-3-O$ - $\beta$ - D - glucos, $(B)$ compound(2), quercet $\in$ - 3-methoxy-3-0-(4acetylrhamnoside)-7-0-a-rhamnoside, (C) Compound (3),kaempferol-4'-methoxy-3,7-0-dirhamnoside. 
A)

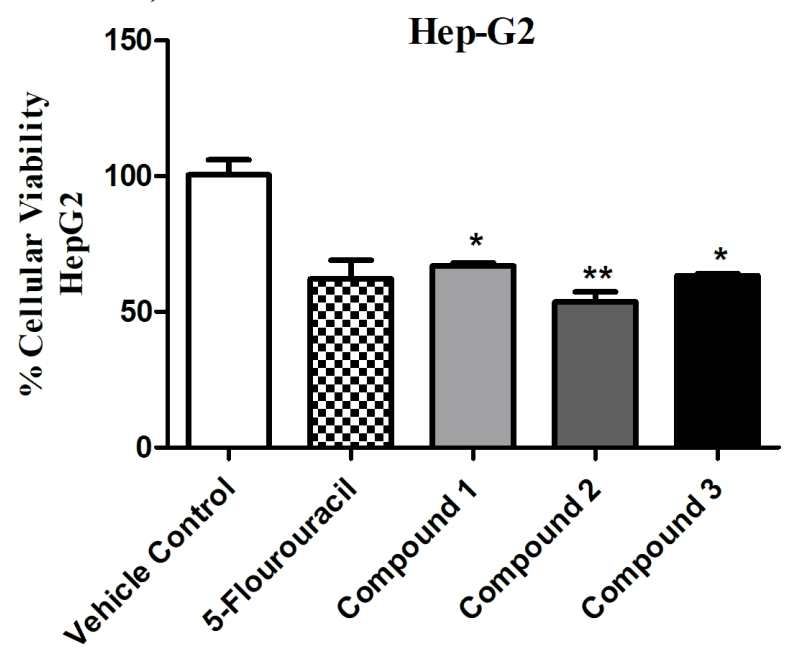

C)

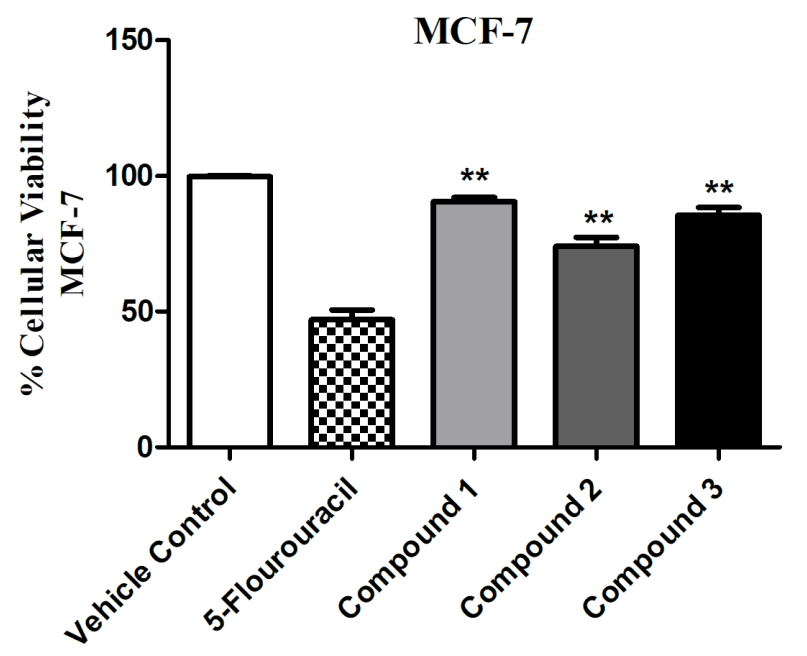

B)

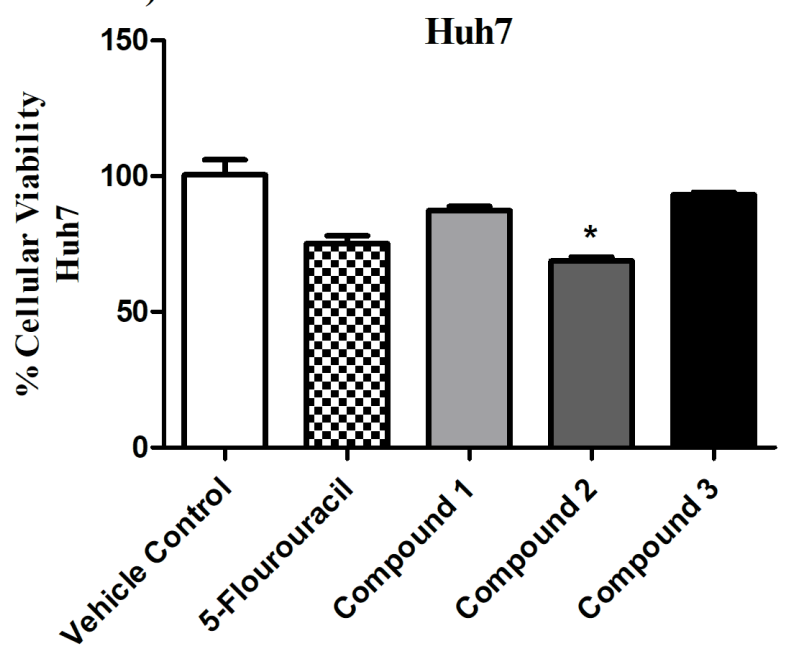

D)

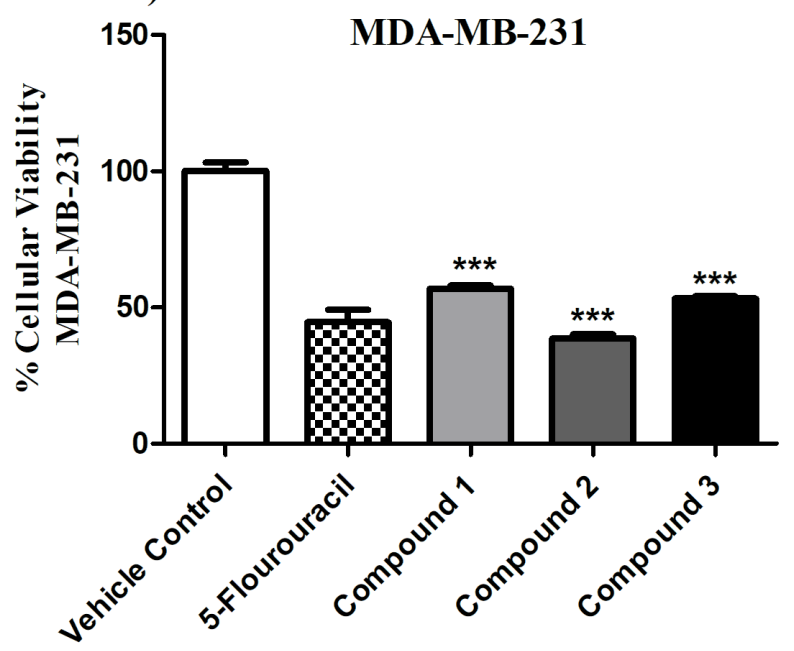

Figure 2

Preferntial selectivity of flavonol glycosides towards MDA-MB-231 TNBC cell lines The impact of Compounds (1-3) at a concentration $20 \mu \mathrm{M}$ on cellular viability of different cancer cell lines was assessed using MTT assay, where treated cells were compared to cells treated with vehicle only (Vehicle control wells). 5-Flourouracil was used as a positive control in this study. Compounds (1-3) showed cytotoxic effects against (A) MDA-MB-231, (B) MCF-7, (C) Hep-G2 and (D) Huh-7 cells with a preferential selectivity and more pronounced effects against MDA-MB-231. Student $t$ test was performed. Data are presented as mean \pm SEM of three independent experiments; $* * *=P<0.001, * *=P<0.01, *=P<0.05$ compared with control group. 


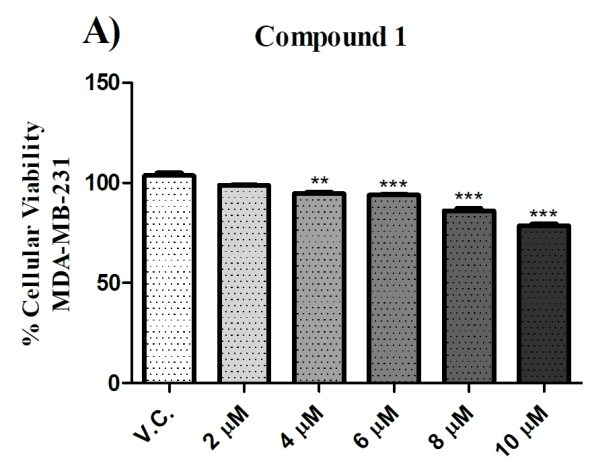

B)

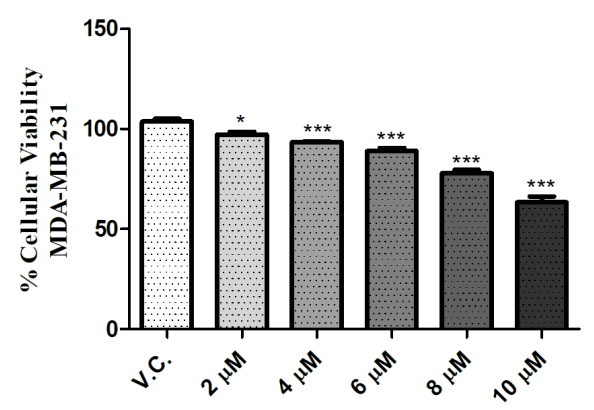

E)

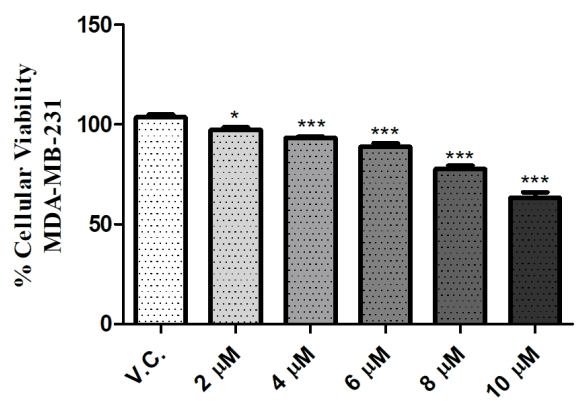

C) Compound 3

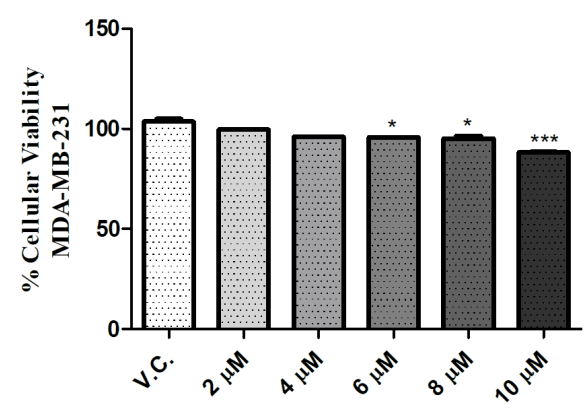

F)

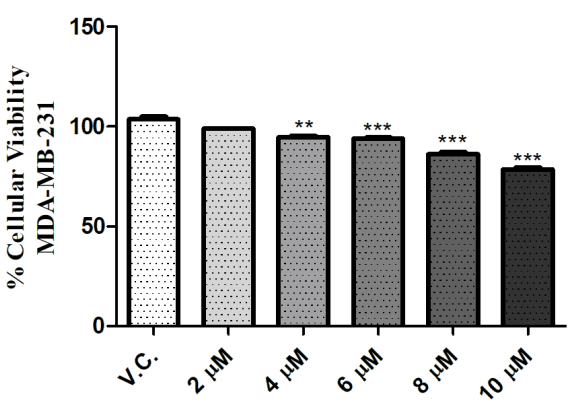

Figure 3

Concentration dependent effect of flavonol glycosides isolated from C. droserifolia on MDA-MB-231 cellular viability and proliferation rates Cellular viability of MDA-MB-231 was measured using MTT assay. The \% viability of cells were calculated relative to cells treated vehicle control, serial dilutions of Compound 1 (A) (D), Compound 2 (B) (E) and Compound 3 (D) (F) resulted in a concentration dependent reduction in MDA-MB-231 cellular viability and proliferation rate. Student $t$ test was performed. Data are presented as mean \pm SEM of three independent experiments; ${ }^{\star \star \star}=P<0.001,{ }^{*}=P<0.01,{ }^{*}=P<0.05$ compared with control group. 

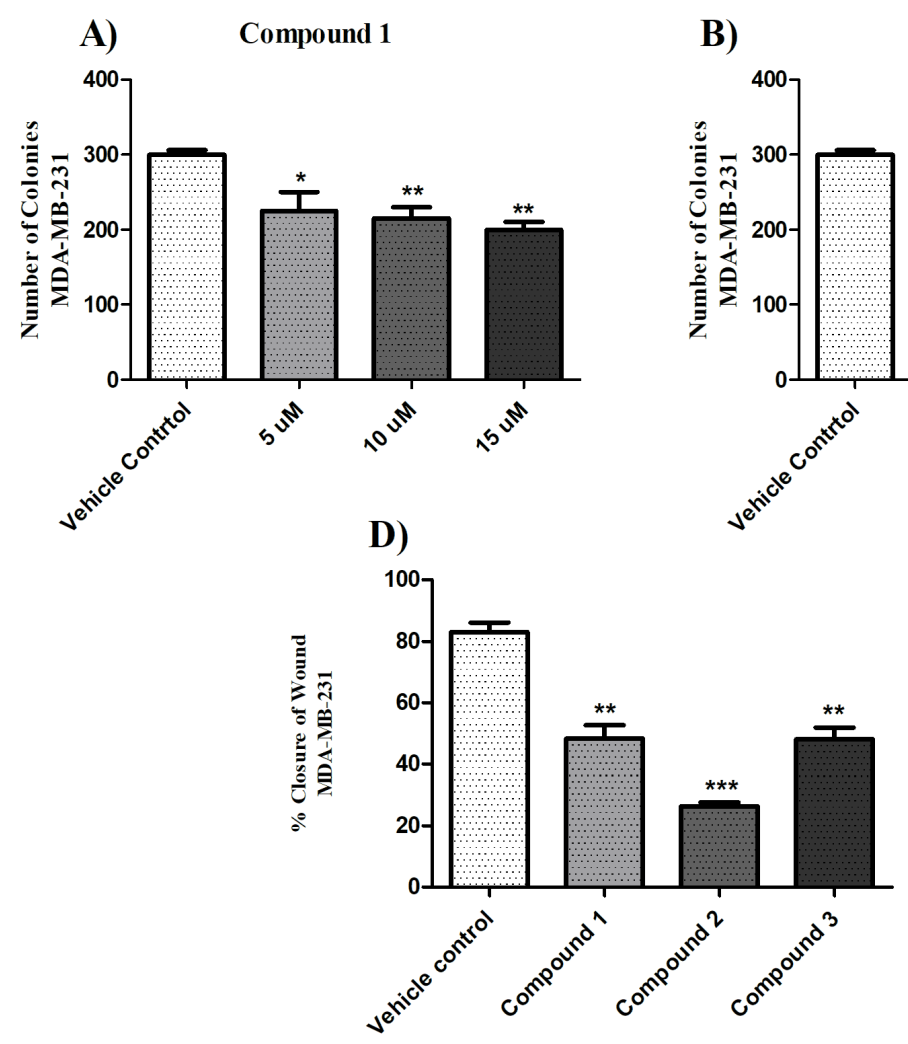
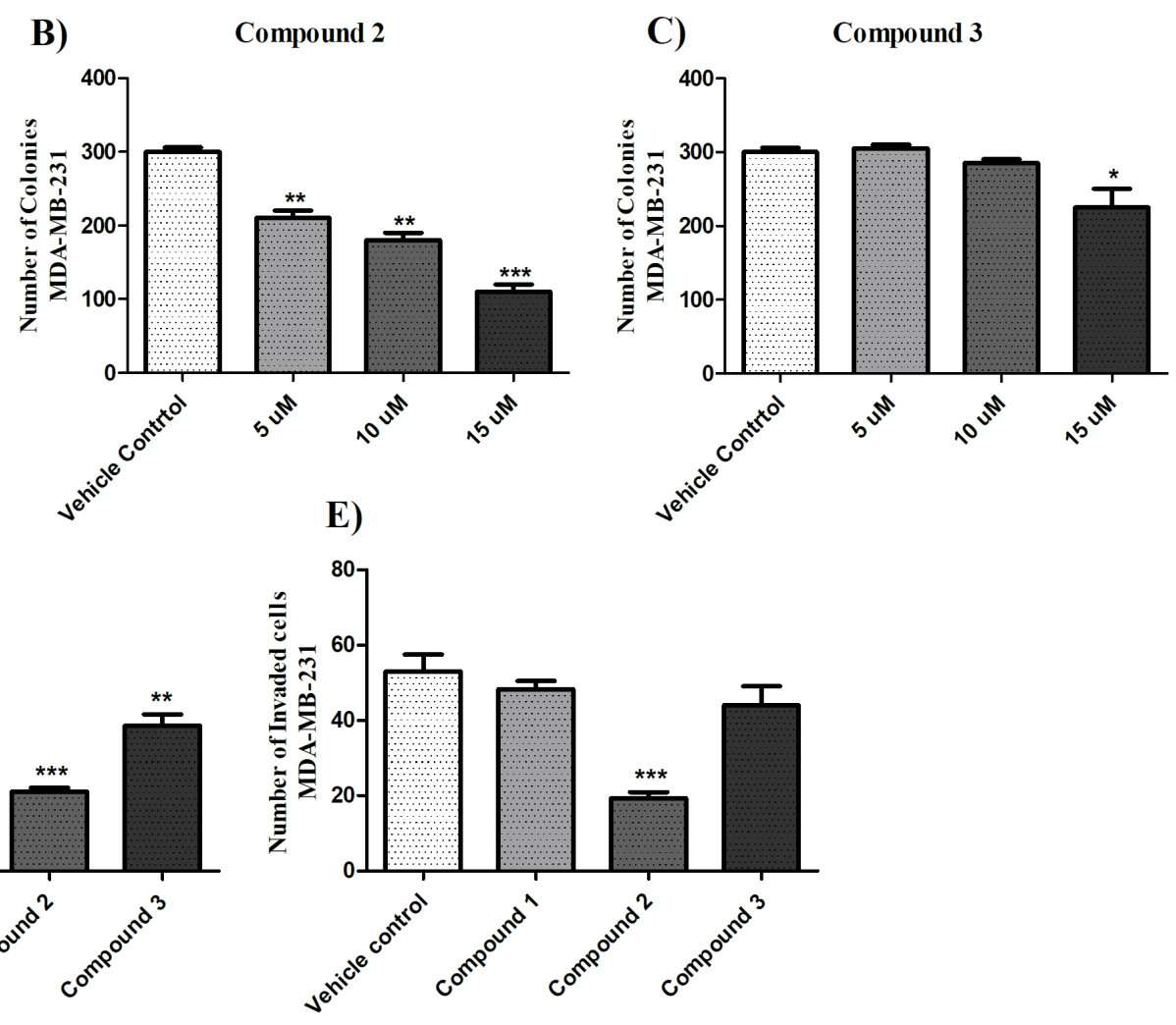

\section{Figure 4}

Impact of flavonol glycosides isolated from C. droserifolia on colony forming ability, migration, and invasion capacity of MDA-MB-231 cell lines The impact of compounds (1-3) on MDA-MB-231 colony forming ability, migration ability and invasion capacity were assessed using colony forming assay, scratch assay and modified boyden chamber assay, respectively. (A) Compound 1, (B) Compound 2, and (C) Compound 3 showed a concentration dependent reduction in MDA-MB-231 clonogenicity compared to vehicle control cells. Also Compounds (1-3) showed a repressing ability of MDA-MB-231 migration ability (D) while only compound 2 showed a significant repression of MDA-MB-231 invasion capacity (E). Student $t$ test was performed. Data are presented as mean \pm SEM of three independent experiments; $* \star \star=$ $P<0.001,{ }^{*}=P<0.01, *=P<0.05$ compared with control group. 

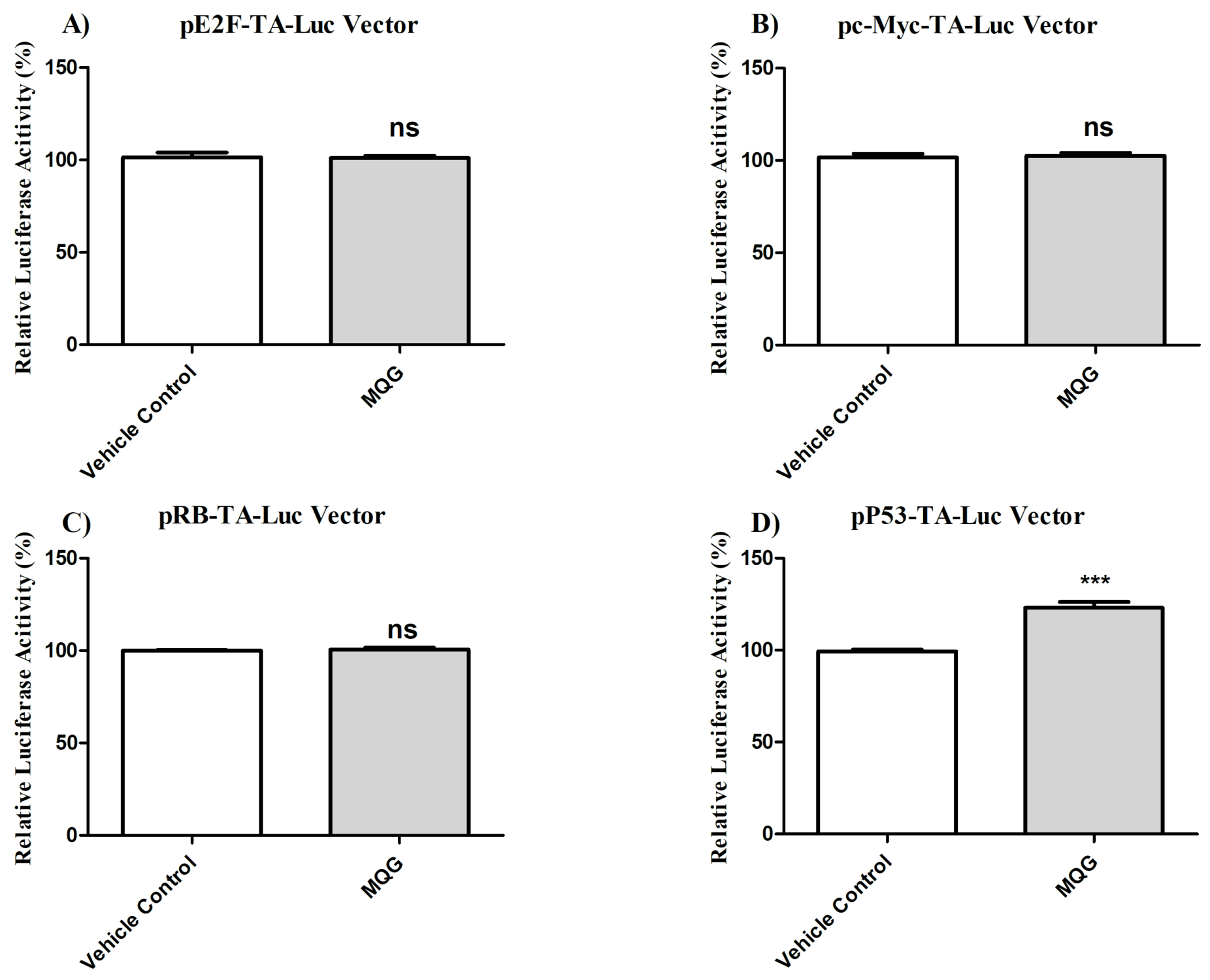

Figure 5

Impact of Compound 2 (MQG) on vital cell cycle regulators MDA-MB-231 cells treated with compound 2 (MQG) resulted in (a) significant induction of TP53 luciferase activity. However, it showed non-significant effects on (b) cMYC (c) RB and (d) E2F luciferase activity. Data are presented as mean \pm SEM of three independent experiments; ${ }^{* \star} p<0.001,{ }^{* \star} p<0.01$ compared with mock cells. miRNA: microRNA; SEM: standard error of the mean; siRNA: small interfering RNA 
A) MDA-MB-231

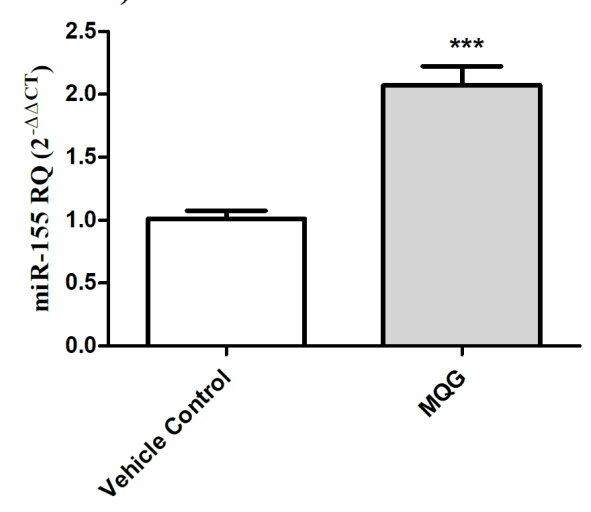

B)

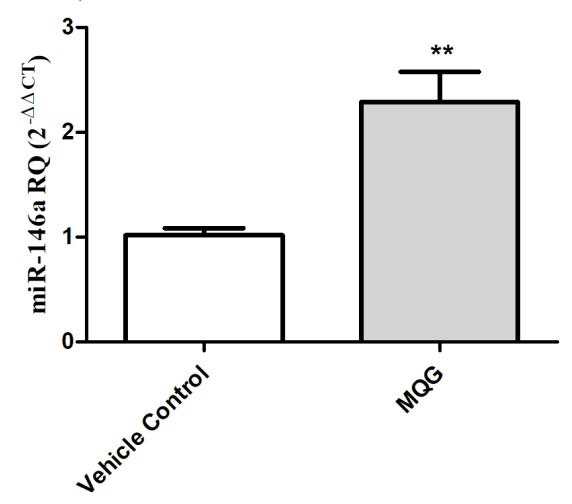

MDA-MB-231

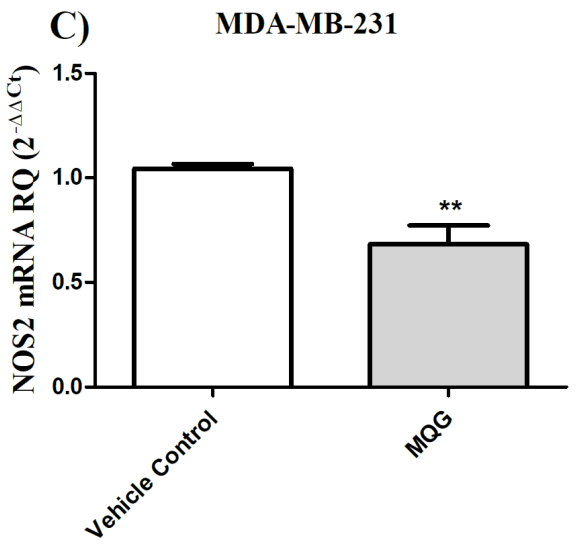

D) MDA-MB-231

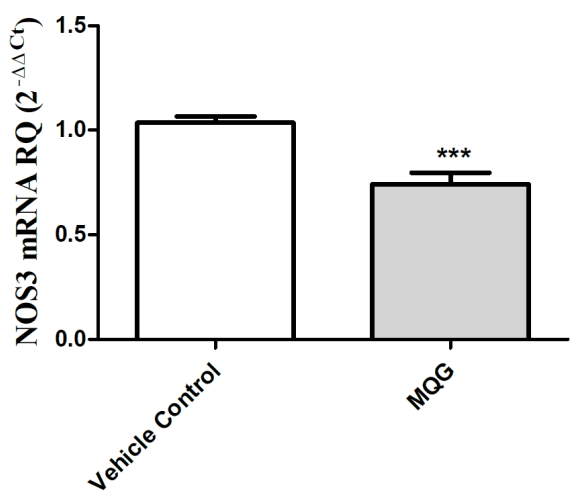

E) MDA-MB-231

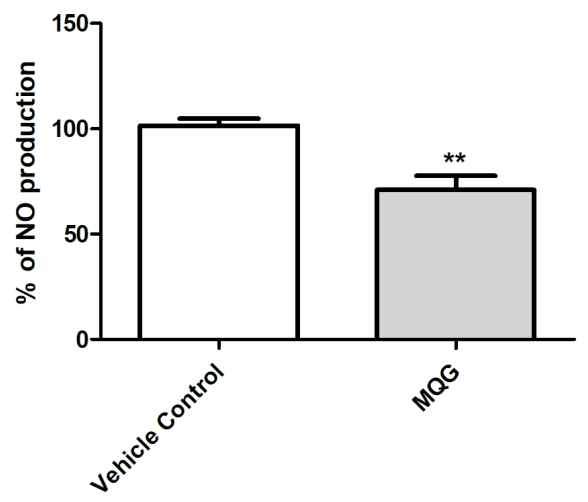

\section{Figure 6}

Impact of Compound 2 (MQG) on the ceRNA circuit MALAT-1/miR-155/miR-146a in TNBC cell line, MDAMB-231. MALAT-1, miR-155 and miR-146a expression levels were assessed in MDA-MB-231 cells using qRT-PCR and normalized to RNU6B (for miRNAs) and $\beta$-actin (for MALAT-1) as internal controls. MDA-MB231 cells were treated with $10 \mu \mathrm{M}$ of Compound 2 (MQG) which resulted in a significant repression of $(A)$ MALAT-1 with a marked elevation of (B) miR-155 (C) miR-146a simultaneously when compared to vehicle control cells. Student $t$ test was performed. Data are presented as mean \pm SEM of three independent experiments; ${ }^{\star \star \star}=P<0.001,{ }^{* *}=P<0.01,{ }^{*}=P<0.05$ compared with control group. 
A)
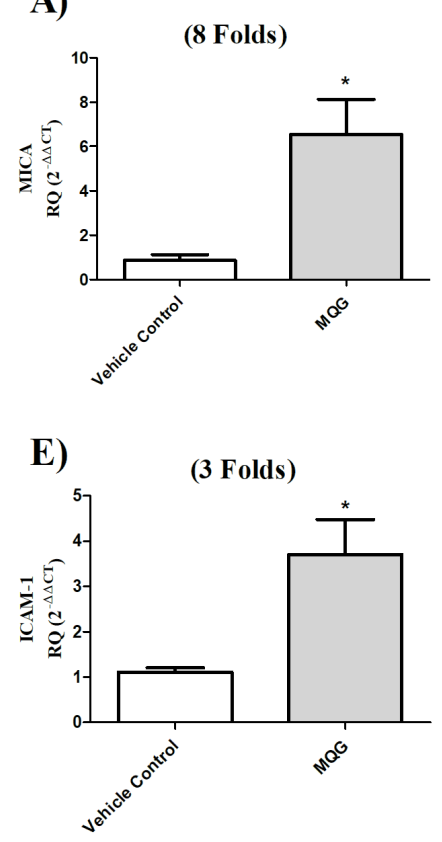

B)

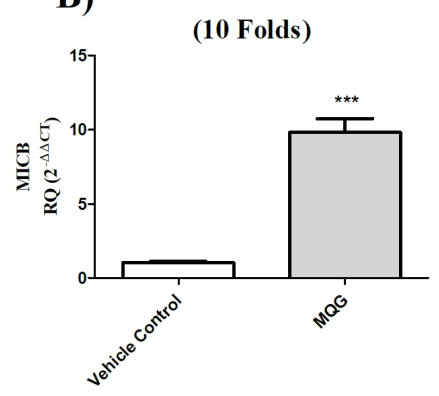

F)

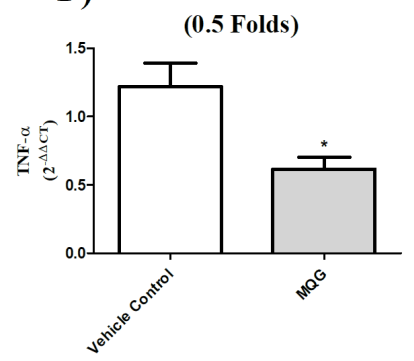

C)

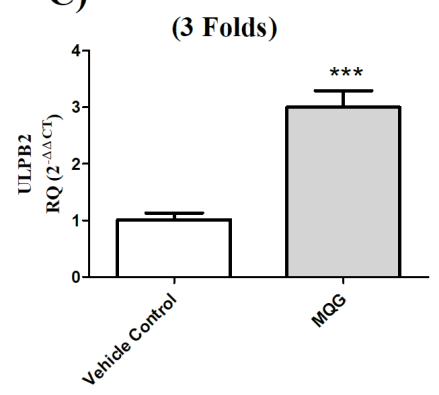

G)

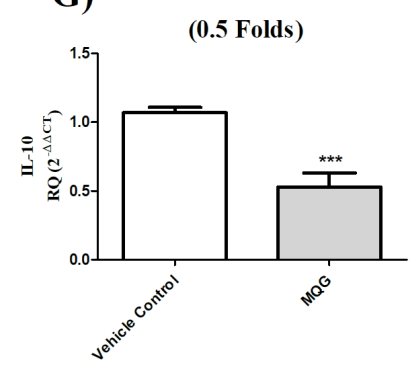

D)

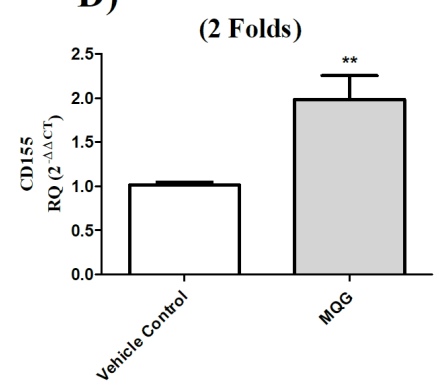

H)

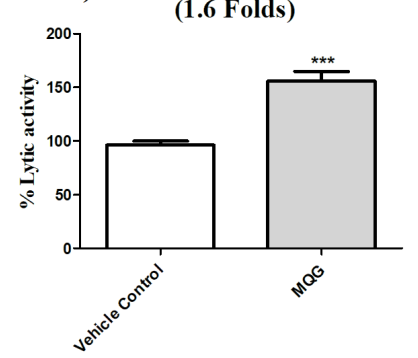

\section{Figure 7}

Impact of Compound 2 (MQG) on the NO machinery in TNBC cell line, MDA-MB-231. NOS2 and NOS3 expression levels were assessed in MDA-MB-231 cells using qRT-PCR and normalized to $\beta$-actin as internal controls. MDA-MB-231 cells were treated with $10 \mu \mathrm{M}$ of Compound 2 (MQG) which resulted in a marked repression of (A) NOS2 and (B) NOS3 mRNA levels when compared to vehicle control cells. Moreover, Compound 2 (MQG) resulted in a significant reduction in (C) NO production in the supernatant of MDA-MB-231. Student $t$ test was performed. Data are presented as mean \pm SEM of three independent experiments; $* \star \star=P<0.001, * \star=P<0.01,{ }^{*}=P<0.05$ compared with control group.

\section{Image not available with this version}

\section{Figure 8}

Impact of Compound 2 (MQG) on NK cells cytotoxicity and tumor microenvironment MICA, MICB, ULBP2, ICAM-1, CD155, TNF- $\alpha$ and IL-10 expression levels were assessed in MDA-MB-231 cells using qRT-PCR and normalized to $\beta$-actin as an internal control. MDA-MB-231 cells were treated with $10 \mu \mathrm{M}$ of 
Compound 2 (MQG) resulted in a marked increase in (A) MICA (B) MICB (C) ULBP2 (D) CD155 (E) ICAM-1 simultaneously with a marked repression of (F) TNF-a and (G) IL-10 transcript levels when compared to vehicle control cells. Moreover, Compound $2(M Q G)$ resulted in a significant induction of $(H)$ primary NK cells cytolytic activity. Student $t$ test was performed. Data are presented as mean \pm SEM of three independent experiments; ${ }^{* \star}=P<0.001,{ }^{* *}=P<0.01,{ }^{*}=P<0.05$ compared with control group.

\section{Image not available with this version}

\section{Figure 9}

A schematic presentation of the molecular mechanisms drawn downstream MQG in TNBC cells This figure represents a summative figure highlighting the molecular mechanisms tackled by MQG in MDAMB-231 TNBC cells. MQG harnesses TNBA progression through repressing MALAT-1/miR-155/miR-146a axis that consequently altered the NO biosynthetic pathway, immune ligands for natural killer cells and cytotoxic T lymphocytes and finally alleviated the immune suppressive nature of tumor microenvironment of TNBC cells 